\title{
e-Migrinter
}

$11 \mid 2013$

Et l'immobilité dans la circulation?

\section{Migrants malgré eux}

Séminaire « doctorants-chercheurs », Migrinter

Eleonora Guadagno, Véronique Lassailly-Jacob et Hélène Simon-Lorière

\section{(2) OpenEdition}

\section{Journals}

Édition électronique

URL : https://journals.openedition.org/e-migrinter/265

DOI : 10.4000/e-migrinter.265

ISSN : 1961-9685

Éditeur

UMR 7301 - Migrinter

Édition imprimée

Date de publication : 1 septembre 2013

Pagination : 188-192

ISSN : 1961-9685

Référence électronique

Eleonora Guadagno, Véronique Lassailly-Jacob et Hélène Simon-Lorière, « Migrants malgré eux 》, eMigrinter [En ligne], 11 | 2013, mis en ligne le, consulté le 20 mai 2021. URL : http://

journals.openedition.org/e-migrinter/265; DOI : https://doi.org/10.4000/e-migrinter.265 
VIE DU LABO

\title{
« Migrants malgré eux ", séminaire « doctorants-chercheurs », Migrinter, Poiters, le 5 décembre 2012
}

\author{
Compte rendu par Eleonora Guadagno, Véronique Lassailly-Jacob \\ \& Hélène Simon-Lorière
}

$\mathbf{L}$

e 5 décembre 2012 s'est tenue la première rencontre des séminaires « doctorantschercheurs » organisés par Migrinter à destination du master " Migrations Internationales ". Cette séance, intitulée « Migrants malgré eux ", a été animée par Eleonora Guadagno et Hélène Simon-Lorière, toutes deux doctorantes à Migrinter, et par Véronique Lassailly-Jacob, géographe, enseignante et chercheure à Migrinter. Fabienne

Le Houérou, historienne et chargée de recherche au CNRS a également contribué à animer le débat en tant que chercheure invitée à Migrinter ce jour-là.
Conçue lors de l'assemblée générale (AG) de Migrinter de juin 2012, la formule du séminaire « doctorant-chercheur » prévoit une analyse de la part d'un ou plusieurs doctorants d'un texte écrit il y a plusieurs années par un chercheur du laboratoire. Ce dernier intervient ensuite pour commenter et replacer de manière critique le texte dans le cadre de ses recherches actuelles.

Eleonora Guadagno et Hélène Simon-Lorière ont choisi un article de Véronique Lassailly-Jacob, «Migrants malgré eux» (Lassailly-Jacob, 1999), pour l'avoir interrogé dans le cadre de leurs recherches sur les migrations forcées : celles dues à l'environnement en Italie pour la première et celles dues à la guerre civile libérienne pour la seconde. Elles ont confronté ce premier texte avec un article d'Anthony Richmond, "Sociological theories of international migration, the case of refugees » (Richmond, 1988). L'attention qu'elles ont portée à ces deux textes est liée à leur réflexion commune sur les définitions et les classifications des catégories de réfugiés et de migrants, mais est aussi liée au fait que les deux articles, qui se réfèrent à un contexte historique bien différent de celui d'aujourd'hui, sont encore des sources d'analyse et d'observation utiles. E. Guadagno a d'abord résumé les apports des deux articles avant qu'ils soient discutés par chaque intervenante du séminaire. 
L'article "Migrants malgré eux» est centré sur la notion de mobilité forcée comme symptôme d'une crise profonde et fuite d'une situation insupportable. Après avoir retracé l'évolution des différentes typologies de migrations forcées, V. Lassailly-Jacob s'interroge sur le "réel" niveau de choix des migrants "volontaires" » et se propose de décloisonner les catégories de migrants forcés et migrants volontaires en considérant que les facteurs de mobilité se mêlent dans de nombreux cas de crises complexes, qu'elles soient politiques, démographiques, économiques et/ou écologiques. Même si la mobilité volontaire semble plus sélective et la mobilité forcée plus collective, elle souligne que les individus peuvent passer d'une catégorie à l'autre. Elle donne l'exemple de familles nomades bergères qui effectuent des parcours saisonniers mais qui peuvent interrompre cette circulation en raison d'une sécheresse et s'installer dans un territoire étranger ou bien qu'elles avaient l'habitude de traverser. Ici, leur transhumance se transforme en migration forcée. Les catégories proposées par les textes des lois internationales appliqués à classer les populations migrantes se révèlent, selon la chercheuse, de plus en plus inadaptés du fait de leurs définitions trop strictes qui cloisonnent, enferment, les populations dans des catégories sans tenir compte du dynamisme du phénomène migratoire.

L'article d'Anthony Richmond retrace l'évolution des définitions des mobilités volontaires et forcées au sein des différentes théories migratoires, en relevant à quel point cette distinction est peu valide lorsqu'on analyse les systèmes globaux dans lesquels déterminants politiques, économiques et environnementaux se mêlent. A. Richmond propose plutôt un continuum d'analyse, c'està-dire une façon dynamique d'étudier un phénomène, pour observer des situations de choix faits pour maximiser les avantages - ce qu'on appellerait des migrations «volontaires» - et de choix faits sous contrainte donnant lieu à des déplacements considérés comme des migrations «forcées». Entre les deux extrêmes s'intercalent de nombreuses situations difficilement classifiables du point de vue du droit international en raison du fait que, dès le début des années 1990, se multiplient les restrictions étatiques à la liberté de mouvement et les dégradations politiques et/ou environnementales des conditions de vie des populations de certaines régions du monde.

H. Simon-Lorière s'est plus particulièrement penchée, dans ces deux articles, sur le double mouvement d'homogénéisation, avec l'idée de continuum et de différenciation suggéré par les deux textes. En effet, les auteurs suivent une démarche de catégorisation scientifique, soulignant le poids des "déterminants de la migration », tout en insistant sur la difficulté à démêler les causes des migrations : cette démarche permet d'interroger la place des individus et des groupes à l'intérieur du phénomène migratoire et celle de la volonté, de la liberté, ou de l'«autonomie», pour reprendre un terme employé par Emmanuel Ma Mung (Ma Mung, 2009) ou Constance de Gourcy (Gourcy, 2005). Cette approche permet de souligner la diversité des situations au sein de ce qu'on aurait identifié comme un seul et même groupe, celui des migrants. Dans son travail de thèse, H. Simon-Lorière réfléchit sur ces catégories et tente d'établir une typologie des réfugiés libériens rencontrés, en s'appuyant notamment sur une dimension incontournable, celle de la temporalité. Elle s'attache également au poids des «stratégies d'acteurs » de ces réfugiés qui sont avant tout des migrants, même si leur statut juridique influe sur les trajectoires observées au moment de la fin du conflit civil au Libéria.

Les deux textes permettent de souligner l'évolution épistémologique qu'ont suivie les études sur les réfugiés depuis les années 1990. Influencées par la catégorisation juridique de la Convention de 
Genève de 1951, ces études ont toujours été proches des organisations humanitaires, dans une logique de recherche-action combinée à une approche critique assumée - voir les travaux des Refugee Studies et notamment de leur fondatrice, Barbara Harrel-Bond ${ }^{1}$. D'abord centrées sur les réfugiés, ces recherches se sont décloisonnées au fil des années 1990 et 2000, s'ouvrant s'abord aux déplacés internes puis aux rapatriés. Cette évolution s'est faite au gré de débats importants entre réalistes et nominalistes: les réalistes considèrent que les expériences des réfugiés sont fondamentalement spécifiques par rapport à celles des migrants dits «volontaires» et ne doivent pas être diluées dans les mouvements de la dynamique migratoire mondiale. Les réfugiés ont en effet un statut qui les protège et qui ne devrait pas être remis en cause par des recherches les assimilant aux migrants. D'un autre côté, les nominalistes soulignent que la catégorie de réfugié, construite et restrictive, pourrait englober davantage de situations et renvoie à des expériences parfois proches de celles des autres migrants. Adoptant plutôt cette dernière approche, les études sur les réfugiés se sont peu à peu rattachées aux recherches sur les migrations en général, évoluant vers la dénomination de Forced Migration Studies.

Dans sa thèse sur les réfugiés libériens à Conakry, H. Simon-Lorière prend acte de cette évolution et s'empare de l'idée d'un continuum d'analyse pour appréhender les expériences migratoires de ces réfugiés dans leur complexité spatiale et temporelle. En reconstituant leurs parcours migratoires, elle a observé qu'un seul individu avait pu passer d'une catégorie à une autre, de la même manière qu'il pouvait jouer sur des identités et des statuts juridiques divers pour mettre en œuvre des projets migratoires depuis la fin du conflit civil. Néanmoins, le statut de réfugié joue un rôle important dans ces projets car il donne accès à des programmes

\footnotetext{
1 Voir notamment Harrell-Bond, Barbara (1986)
} Imposing Aid, Oxford, Oxford University Press, 480 p. spécifiques pour rentrer «au pays », mais surtout pour partir légalement vers des pays plus riches.

Dans son travail doctoral, E. Guadagno analyse le cas de la population de Sarno, une ville située près de Naples en Italie, que la population a dû quitter pour se réinstaller soit dans d'autres communes, soit dans d'autres quartiers de Sarno moins touchés par le glissement de terrain qui l'a frappée en 1998. E. Guadagno propose de déconstruire les catégories proposées par l'article de V. Lassailly-Jacob, c'est-à-dire que des mobilités forcées peuvent devenir des mobilités volontaires, ou être accompagnées par ce type de déplacement. En effet, dans le cas du glissement de terrain de Sarno, elle a observé que la majorité des personnes s'étaient déplacées pour une raison exogène, mais que d'autres personnes avaient suivi ce mouvement sans avoir été touchées directement par la catastrophe. Il s'agit là d'une migration volontaire, qui contribue à interroger la dichotomie entre migrations forcées et volontaires, qui pourraient plutôt être considérées comme les deux faces d'une même pièce. La réalité des expériences individuelles est en effet de moins en moins assignable à des catégories figées. Pourtant, les définitions juridiques s'appuient sur ces catégories qui permettent à la fois de protéger certains individus, ou, bien au contraire, de ne pas leur venir en aide. Il est désormais impératif, pour toute tentative de classification scientifique, de parvenir à s'affranchir de ces catégories juridiques nationales et internationales de plus en plus restrictives, afin d'analyser les expériences migratoires actuelles.

Les facteurs de mobilité volontaire ou contrainte, confondus dans les motivations personnelles et les projets individuels, se superposent à un contexte d'obligations et de contraintes externes croissantes (contrôle aux frontières, papiers et taxes de circulation) qui modifient l'espace et la 
temporalité des parcours migratoires. Les causes des déplacements sont en effet multiples et mélangées: de nature économique, écologique, politiques, etc. En ce sens, l'article d'A. Richmond confirme que l'opposition entre migrations forcées et volontaires est un anachronisme, voire un postulat non pertinent pour la recherche. Dans le cas des expériences migratoires analysées à l'issue du terrain mené en Italie, le continum proposé par A. Richmond semble être utile pour analyser les causes de la migration qui allient causes coercitives externes et nécessités endogènes dans un contexte caractérisé à la fois par des asymétries de pouvoir, une catastrophe environnementale et une crise économique.

Selon V. Lassailly Jacob, ces textes questionnent tous deux la mobilité forcée et les catégories juridiques et scientifiques existantes, mais ils ont été produits dans deux contextes intellectuels distincts, l'un français et l'autre anglo-saxon et qui, pour des raisons politiques internationales (notamment le colonialisme et la Guerre froide) ont toujours abordé la question des mobilités forcées avec des approches très différentes. Ils sont aussi le reflet des années 1990, qui diffèrent du contexte actuel, tant en ce qui concerne les institutions internationales et les politiques migratoires que les recherches sur les migrations. Elle souligne que de nombreux cas de migrations sous contrainte n'ont pas obtenu de reconnaissance juridique internationale par le passé. La géographe continue de penser que les catégories juridiques ne permettent pas de décrire la richesse des réalités individuelles, elles-mêmes en perpétuel mouvement: une même personne peut en effet connaitre des statuts juridiques divers selon ses expériences de mobilité.

Le séminaire s'est clôt sur une série d'échanges entre Fabienne Le Houérou et les participants, doctorants du laboratoire Migrinter et étudiants du master
"Migrations internationales » autour de la déconstruction des catégories par les pratiques de terrain et de recherche, mais également à propos de l'influence des définitions figées sur ces mêmes pratiques ainsi que sur les discours. Ces définitions apparaissent en effet bien cruelles pour tous ceux qui, expérimentant pourtant une forme de mobilité, ne rentreraient pas dans les critères figés des définitions juridiques et/ou scientifiques établies jusqu'alors et concentrées essentiellement sur la protection des réfugiés, et qui se trouveraient ainsi privés de toute forme de protection.

Eleonora Guadagno Doctorante en Géographie Migrinter - UMR 7301

CNRS / Université de Poitiers guadagno.eleonora@gmail.com

Véronique Lassailly-Jacob Professeur de géographie Université de Poitiers / CNRS Migrinter - UMR 7301 vlassail@univ-poitiers.fr

Hélène Simon-Lorière Doctorante en Géographie Migrinter - UMR 7301

CNRS / Université de Poitiers hsimonloriere@gmail.com 
Bibliographie :

Gourcy, Constance de (2005) L'autonomie dans la migration. Réflexion autour d'une énigme, Paris, L'Harmattan, 348 p. (Logiques Sociales).

Harrell-Bond, Barbara (1986) Imposing Aid, Oxford, Oxford University Press, 480 p.
Ma Mung, Emmanuel (2009) Le point de vue de l'autonomie dans l'étude des migrations internationales, in Dureau F., Hily M.-A., Les mondes de la mobilité, Rennes, Presses de l'Université de Rennes, pp. 25-38.

Les articles analysés lors de ce séminaire sont consultables à partir du lien ci-dessous :

Lassailly-Jacob, Véronique (1999) Migrants malgré eux. Une proposition de typologie, in Lassailly-Jacob V. ; Marchal J.-Y., Quesnel A. (éd.) Déplacés et réfugiés : la mobilité sous contrainte, Paris, IRD, pp. 27-48. [En ligne] URL:

http://horizon.documentation.ird.fr/exl-doc/pleins textes/divers4/010017907.pdf

Richmond, Anthony (1998) Sociological theories of international migration, the case of refugees, Current Sociology Vol. 36, N², pp. 7-25. [En ligne] URL :

http://yorkspace.library.yorku.ca/xmlui/bitstream/handle/10315/8016/Current\%20Sociology-1988- 